\title{
New contributions on line-projections in omnidirectional vision
}

\author{
Jesus Bermudez-Cameo* \\ * Dept. Informtica e Ingeniera de Sistemas, I3A - Universidad de Zaragoza, Spain \\ Advisors: Jos Jess Guerrero and Gonzalo Lpez-Nicols \\ Date and location of PhD thesis defense: February 4, 2016, Universidad de Zaragoza
}

Received 29st June 2016; accepted 24st July 2016

\begin{abstract}
Computer vision has an increasing interest in most fields of emerging technologies. A challenging topic in this field is to study how to enlarge the field of view of the camera systems to obtain more information of the environment in a single view. In particular, omnidirectional vision can be useful in many applications such as estimating location in robotics, autonomous driving and unmanned aerial vehicles. The wide field of view of omnidirectional cameras allows taking advantage of describing 3D scenarios using line features. On the one hand, line features represent natural landmarks in man-made environments, they are easy to understand, coincident with edges of constructive elements and often still present when having texture-less scenarios. On the other hand, long segments are especially useful for drift compensation because they are usually completely visible on the omnidirectional projection. However, in omnidirectional cameras line projections are distorted by the projection mapping becoming complex curves.

This thesis * is focused on the geometry of line projections (line-images) in omnidirectional systems. Main addressed topic of this work is line-image extraction on different kinds of central and non-central omnidirectional images. However, due to the nature of projection in omnidirectional cameras, other addressed topics are camera calibration and, in the case on non-central cameras, 3D reconstruction from single images.

In particular, we analyzed the following problems involving line projections in central and non-central omnidirectional cameras (see Fig. 1):
\end{abstract}

Line-image extraction in central omnidirectional cameras. The presented research begins with line-image extraction from hypercatadioptric images which is used for estimating dominant directions in Manhattan scenarios $[1,2,3]$. This extraction approach has been generalized to a framework for central imaging systems obtaining the equations of line-images curves for a set of different catadioptric and dioptric systems [4]. Using this framework we have developed the plumb-line constraint for each of these systems and obtained analytical solutions that allow simultaneously recovering camera calibration and line-images [5,6]. We have integrated these theoretical solutions in an automatic method for line-image extraction in central systems when the calibration is unknown. This proposal is compiled in a fully functional toolbox ${ }^{\dagger}$ which has been released as open

\footnotetext{
Correspondence to: <bermudez@unizar.es>

Recommended for acceptance by David Vázquez Bermúdez

ELCVIA ISSN:1577-5097

DOI:http://dx.doi.org/10.5565/rev/elcvia.948

Published by Computer Vision Center / Universitat Autònoma de Barcelona, Barcelona, Spain

${ }^{*}$ webdiis.unizar.es/\%7Ebermudez/bermudezCameoThesis.pdf

${ }^{\dagger}$ webdiis.unizar.es/\%7Ebermudez/toolbox.html
} 
source for research purposes.

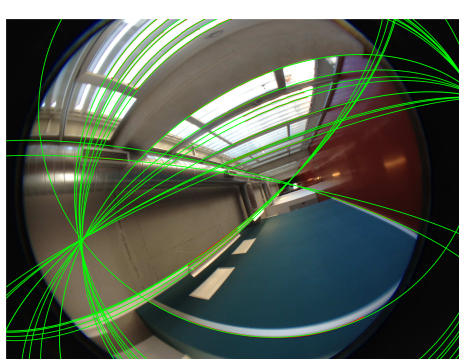

(a)

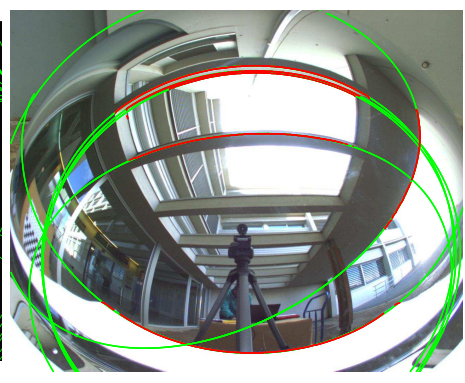

(b)

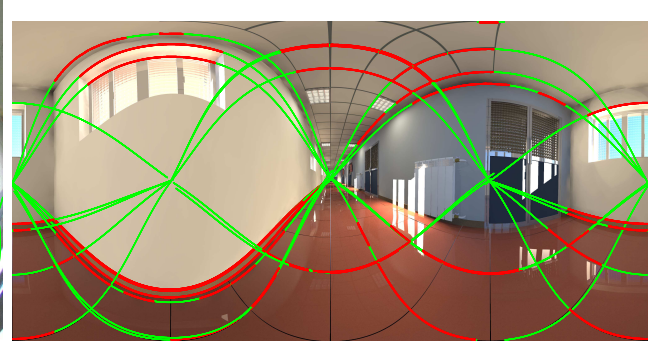

(c)

Figure 1: Extraction of line projections in different omnidirectional images:

(a) central fisheye.

(b) non-central spherical

(c) non-central panorama.

Line-image extraction and 3D line fitting in non-central omnidirectional cameras. The characteristics of the projection in non-central systems allow recovering the complete 3D information of a line from a single projection. The non-central systems addressed in this thesis are the conical and spherical catadioptric systems (easy to manufacture and without singular central configurations) and the non-central circular panorama. Generalizing the description used for central systems we have developed the analytical line-image equations for conical and spherical catadioptric systems. The particularization for conical catadioptric systems allows us to recover the geometry of the mirror and the geometry of the 3D line from five points of the line-image. Main problem of recovering 3D from single projections in non-central systems is that accuracy is very noise sensitive and that line-image extraction is a challenging unsolved task since four degrees of freedom are involved. To address this issue we propose different solutions and approaches. To measure the accuracy we introduce the concept of effective baseline among a set of rays which strongly depends on the class of non-central system. Regarding accuracy in line 3D fitting the non-central panorama is the system obtaining the best results although its constructive difficulties limits its use in practice. To solve the problem of automatic line-image extraction in non-central systems we propose a random robust approach using a pre-evaluation step, similar to the one used in PROSAC, and using different kind of distances. In particular, we have analytically solved the Euclidean distance from a point to line-image for conical [7] and spherical catadioptric systems [8] and we propose a distance based on the projection of the closest points in the 3D space for the non-central circular panorama [9].

Minimal solutions for line-image fitting in non-central cameras by imposing geometric constraints. Another way to increase the accuracy and the stability of line reconstruction is exploiting additional geometric constraints and using prior information. In this thesis, we propose a set of new minimal solutions for imposing additional constraints between pairs of lines in non-central systems. In particular, we have developed the cases of intersecting orthogonal lines and pairs of parallel lines [10]. Finally we present the minimal solution for computing a line which is parallel to a given plane which can be exploited using the prior information provided by an accelerometer in a Manhattan scenario [11]. 


\section{References}

[1] L. Puig, J. Bermudez, and J. J. Guerrero, "Self-orientation of a hand-held catadioptric system in man-made environments," IEEE International Conference on Robotics and Automation pp:2549-2555, 2010.

[2] J. Bermudez, L. Puig, and J. J. Guerrero, "Line extraction in central hyper-catadioptric systems," OMNIVIS - 10th Workshop on Omnidirectional Vision, Camera Networks and Nonclassical Cameras, 2010.

[3] J. Bermudez-Cameo, L. Puig, and J. J. Guerrero, "Hypercatadioptric line images for 3D orientation and image rectification," Robotics and Autonomous Systems, vol. 60, no. 6, pp. 755-768, 2012.

[4] J. Bermudez-Cameo, G. Lopez-Nicolas, and J. J. Guerrero, "A unified framework for line extraction in dioptric and catadioptric cameras," in 11th Asian Conference on Computer Vision. Lecture Notes in Computer Science., vol. 7727, 2012.

[5] J. Bermudez-Cameo, G. Lopez-Nicolas, and J. J. Guerrero, "Line extraction in uncalibrated central images with revolution symmetry," in 24th British Machine Vision Conference, 2013.

[6] J. Bermudez-Cameo, G. Lopez-Nicolas, and J. J. Guerrero, "Automatic line extraction in uncalibrated omnidirectional cameras with revolution symmetry," International Journal of Computer Vision, vol. 114, no. 1, pp. 16-37, 2015.

[7] J. Bermudez-Cameo, G. Lopez-Nicolas, and J. J. Guerrero, "Line-images in cone mirror catadioptric systems," in 22th International Conference on Pattern Recognition, pp. 2083-2088, 2014.

[8] J. Bermudez-Cameo, , G. Lopez-Nicolas, and J. J. Guerrero, "Fitting line projections in non-central catadioptric omnidirectional systems,", Technical Report, 2015.

[9] J. Bermudez-Cameo, O. Saurer, G. Lopez-Nicolas, J. J. Guerrero, and M. Pollefeys, "Exploiting line metric reconstruction from non-central circular panoramas,", Technical Report, 2015.

[10] J. Bermudez-Cameo, J. P. Barreto, G. Lopez-Nicolas, and J. J. Guerrero, "Minimal solution for computing pairs of lines in non-central cameras," in The 12th Asian Conference on Computer Vision. Lecture Notes in Computer Science, vol. 9003, pp. 585-597, 2014.

[11] J. Bermudez-Cameo, C. Demonceaux, G. Lopez-Nicolas, and J. J. Guerrero, "Line reconstruction using prior constraints in single non-central view,"in 27th British Machine Vision Conference, 2016. 